\title{
ISM simulations: an overview of models
}

\author{
M. A. de Avillez ${ }^{1,2}$, D. Breitschwerdt ${ }^{2}$, A. Asgekar ${ }^{3}$ and E. Spitoni ${ }^{1}$ \\ ${ }^{1}$ Dept. of Mathematics, University of Évora, 7000 Évora, Portugal \\ email: mavillez, spitoni@galaxy.lca.uevora.pt \\ ${ }^{2}$ Dept. of Astronomy \& Astrophysics, Technical University Berlin, D-10623 Berlin, Germany \\ email: breitschwerdt@astro.physik.tu-berlin.de \\ ${ }^{3}$ ASTRON, P.O. Box 2, 7990 AA Dwingeloo, The Netherlands
}

\begin{abstract}
Until recently the dynamical evolution of the interstellar medium (ISM) was simulated using collisional ionization equilibrium (CIE) conditions. However, the ISM is a dynamical system, in which the plasma is naturally driven out of equilibrium due to atomic and dynamic processes operating on different timescales. A step forward in the field comprises a multi-fluid approach taking into account the joint thermal and dynamical evolutions of the ISM gas.
\end{abstract}

Keywords. ISM: general, ISM: structure, atomic processes, turbulence; MHD

\section{Introduction}

The attempts to model the supernova-driven ISM can be traced to the seminal models of Cox \& Smith (1974; CS74) and McKee \& Ostriker (1977, MO77). In the former supernovae (SNe) maintain an interconnected tunnel network filled with X-ray emitting gas, while in MO77 the gas is distributed into three phases in global pressure equilibrium. In both models the Galactic volume (50\% in CS74 and 70-80\% in MO77) is filled with hot $\left(>10^{5} \mathrm{~K}\right)$ low-density gas. Further ramifications include the break-out of the hot intercloud medium, cooling and condensing into clouds (galactic fountain; Shapiro \& Field 1976) or escaping as a wind (e.g., chimney model; Norman \& Ikeuchi 1989).

Although these early works capture some of the essential physics, more complex and sophisticated models were devised by taking advantage of numerical simulations. These comprise the evolution of a patch of the Galactic disk in two dimensions (2D) (hydrodynamical (HD): Bania \& Lyon 1980; Chiang \& Prendergast 1985; Chiang \& Bregman 1988; Rosen et al. 1993; Magnetohydrodynamical (MHD): Vazquez-Semadeni et al. 1995), and in three-dimensions (3D), e.g.. the MHD evolution of a $200^{3} \mathrm{pc}^{3}$ region (Balsara et al. $2004)$ and the cosmic-rays driven amplification of the field in a differentially rotated domain $\left(0.5 \times 1 \times[-0.6,0.6] \mathrm{kpc}^{3} ;\right.$ Hanasz et al. 2004). The first disk-halo evolution models (2D HD) were developed by Rosen \& Bregman (1995). With increasing of computer power, 3D HD (de Avillez model in 2000 and upgrades - see Avillez \& Breitschwerdt 2007; Joung \& Mac Low 2006) and MHD (Korpi et al. 1999; Avillez \& Breitschwerdt 2005; Gressel et al. 2008; Hill et al. 2012) models have been developed.

In general the disk-halo models consider parameters according to observations (e.g., initial matter distribution with height, SN rates, background UV radiation field). Differences are found in the number of physical processes included (magnetic fields, cosmic rays, heat conduction, etc.), numerical techniques, type of grid (fixed or differentially rotated using the shear box technique), and grid resolutions and sizes. Resolutions are fixed or benefit from the use of the adaptive mesh refinement (AMR) technique (Berger \& Oliger 1984). The highest resolutions cover a wide range from 0.5 pc to $10 \mathrm{pc}$, passing through 2 and $8 \mathrm{pc}$. The grid sizes in the vertical direction range from $0.1 \mathrm{kpc}$ to $15 \mathrm{kpc}$ 
on either side of the Galactic midplane. However, grids extending up to $2 \mathrm{kpc}$ imply that the disk-halo-disk cycle can neither be established nor tracked - the simulations are valid for a small period of time before the gas escapes from the top and bottom boundaries.

These simulations showed that: (i) the ISM does not become saturated by SN activity, (ii) the disk expands and relaxes dynamically as SN rate fluctuates in time and space, (iii) the turbulent field builds up exponentially within 20 Myr of disk evolution, (iv) the magnetic field does not strongly correlate with density, except for the densest regions, (v) the magnetic field does not prevent the matter escape into the halo as it only briefly delays the disk-halo cycle, (vi) the volume filling factor of hot gas in the Galactic disk is only $\sim 20 \%$, (vii) there are large pressure variations in the disk in contrast to MO77 with the thermal pressure dominating at high temperatures $\left(T>10^{6} \mathrm{~K}\right)$, magnetic pressure at $T<200 \mathrm{~K}$, and ram pressure elsewhere.

\section{Thermal and dynamical evolution of the ISM}

All models referred previously assumed the ISM plasma to be in CIE, represented by a unique and general cooling function (CF) taken from different sources (e.g., Dalgarno \& McCray 1972 (DM72); Sutherland \& Dopita 1993; Gnat \& Sternberg 2007). CIE assumes that the number of ionizations is balanced by recombinations from higher ionization stages. However, CIE is only valid provided the cooling timescale $\left(\tau_{\text {cool }}\right)$ of the plasma is larger than the recombination times scales of the different ions $\left(\tau_{\text {rec }}^{Z, z}\right)$, something that occurs at $T>10^{6} \mathrm{~K}$ (see references above). For lower temperatures $\tau_{\text {cool }}<\tau_{\text {rec }}^{Z, z}$, and deviations from CIE are expected (see, e.g., DM72). These departures affect the local cooling, which is a time-dependent process that controls the flow dynamics, feeding back to the thermal evolution by a change in the density and internal energy distribution, which in turn modifies the thermodynamic path of non-equilibrium cooling.

A major improvement in ISM studies is therefore to carry out time-dependent multifluid calculations of the joint thermal and dynamical evolution of the plasma, i.e. to follow each fluid element's thermal history by determining its ionization structure and CF at each time-step. Radiative losses are folded into the energy equation with the internal energy including also the potential energies associated to the different ionization stages.

Historically, there have been a number of simulations, which have included part of the ionization history into HD simulations, (Cox \& Anderson 1982; Innes et al. 1987; Borkowski et al. 1994; Smith \& Cox 2001; among others), misty tailored for specific astrophysical problems. The effect of delayed recombination has been emphasized by Breitschwerdt \& Schmutzler (1994), who have modelled the soft X-ray background. Melioli et al. (2009), following the formation and evolution of Hi clouds, only considered the time evolution of selected ions (HI, HII , CII-CiV, and OI-OIII) for temperatures below $10^{6} \mathrm{~K}$, using a fit to the Sutherland \& Dopita (1993) CF for $T \geqslant 10^{6} \mathrm{~K}$. This setup has severe implications in the cooling of the gas as their calculation does not trace the relevant ions recombining to CIV, and OIII .

Recently, owing to the development of the Atomic+Molecular Plasma Emission Code (EA+MPEC) and its coupling to a PPM based AMR code, it has been possible to carry out multi-fluid calculations of the ISM tracing both the thermal and dynamical evolutions of the gas self-consistently. The ionization structure, cooling and emission spectra of $\mathrm{H}$, He, C, N, O, Ne, Mg, Si, S, and Fe ions (with solar abundances; Asplund et al. 2009) are traced on the spot at each time step assuming an equal Maxwellian temperature for electrons and ions (see details and references in Avillez \& Breitschwerdt 2012).

These simulations showed several interesting effects: (i) in a dynamic ISM, the ionization structure and, therefore, the $\mathrm{CF}$, varies with space and time, depending on the 
initial conditions and its history, (ii) the cooling paths in general do not follow the one predicted by static plasma emission calculations, (iii) non-equilibrium ionization X-ray emission in the $\sim 0.25 \mathrm{keV}$ band of gas with $T<10^{5} \mathrm{~K}$ can dominate the corresponding CIE emission at even $T=10^{6.2} \mathrm{~K}$ as a result of delayed recombination, (iv) the presence of Ovi ions at temperatures $<10^{5} \mathrm{~K}$ corresponding to $70 \%$ of the total Ovi mass, and (v) a large fraction of electrons are found in the thermally unstable regime and have a log normal distribution with similar properties (mean and dispersion) to those derived from observations against pulsars with known distances.

\section{Conclusions}

The dynamical and thermal evolution of the ISM are strongly coupled, because the ionization structure determines the $\mathrm{CF}$, which in turn controls the dynamics and thereby the ionization structure, closing a feedback loop. Consequently, strong deviations from CIE occur due to severe mismatches between the different ionization/recombination and dynamical time scales of the plasma. Similar effects due time-dependent cooling are expected in other astrophysical contexts.

\section{Acknowledgements}

M.A.A. thanks the IAU and specifically Y.-H. Chu and V. Reuter for financial support.

\section{References}

Asplund, M., Grevesse, N., Sauval, A. J., \& Scott, P. 2009, ARAA 47, 481

Balsara, D. S., Kim, J., Mac Low, M.-M., \& Mathews, G. J. 2004, ApJ 617, 339

Bania, T. M. \& Lyon, J. G. 1980, ApJ 239, 173

Berger, M. J. \& Oliger, J. 1984, JCP 484

Borkowski, K. J., Sarazin, C. L., \& Blondin, J. M. 1994, ApJ 429, 710

Breitschwerdt, D., Schmutzler T. 1994, Nature 371, 774

Chiang, W.-H. \& Prendergast, K. H. 1985, ApJ 297, 507

Chiang, W.-H. \& Bregman, J. N. 1988, ApJ 328, 427

Cox, D. P. \& Anderson, P. R. 1982, ApJ 253, 268

Cox, D. P. \& Smith, B. W. 1974, ApJ (Letters) 189, L105

de Avillez, M. A. \& Breitschwerdt, D. 2005, A\&A 436, 585

de Avillez, M. A. \& Breitschwerdt, D. 2007, ApJ (Letters) 665, L35

de Avillez, M. A. \& Breitschwerdt, D. 2012, ApJ (Letters) 761, L19

Gnat, O. \& Sternberg, A. 2007, ApJS 168, 213

Gressel, O., Elstner, D., Ziegler, U., \& Rödiger, G. 2008, AËA 486, 35

Hanasz, M., Kowal, G, Otmianowska-Mazur, K., \& Lesch, H. 2004, A\&JA (Letters) 605, L33

Hill, A. S., Joung, M. R., Mac Low, M.-M., et al. 2012, ApJ 750, 104

Innes, D. E., Giddings, J. R., \& Falle, S. A. E. G. 1987, MNRAS 227, 1021

Joung, M. K. R. \& Mac Low, M.-M. 2006, ApJ 653, 1266

Korpi, M. J., Brandenburg, A., Shukurov, A., et al. 1999, ApJ (Letters) 514, L99

Melioli, C., Brighenti, F., D'Ercole, A., \& Gouveia Dal Pino, E. M. 2009, MNRAS 399, 1089

McKee, C. F. \& Ostriker, J. P. 1977, ApJ 218, 148

Norman, C. A. \& Ikeuchi, S. 1989, ApJ 345, 372

Rosen, A., Bregman, J. N., \& Norman, M. L. 1993, ApJ 413, 137

Rosen, A. \& Bregman, J. N. 1995, ApJ 440, 634

Shapiro, P. R. \& Field, G. B. 1976, ApJ 205, 762

Smith, R. K. \& Cox, D. P. 2001, ApJS 134, 283

Sutherland, R. S. \& Dopita, M. A. 1993, ApJ 88, 253

Vazquez-Semadeni, E., Passot, T., \& Pouquet, A. 1995, ApJ 441, 702 